\title{
Congenital Kyphoscoliosis Due to Hemivertebra Treatment Options and Results
}

\author{
Andrés Chahin Ferreyra and Gonzalo Arriagada Ocampo \\ Spine Center Clinica Las Condes \\ University of Chile \\ Chile
}

\section{Introduction}

The clinical impact of congenital vertebral malformations is variable and depends mainly on the type of spinal malformation and the location of this (McMaster \& Ohtsuka, 1982). Defects of formation, segmentation and combination of these presents different rates of progression and final severity of the deformity.

The natural history of congenital kyphoscoliosis has been well documented and shows that most of untreated patients progress to severe deformities (Winter et al., 1968) (Figure 1), and may even present neurological compromise (McMaster \& Singh, 1999).

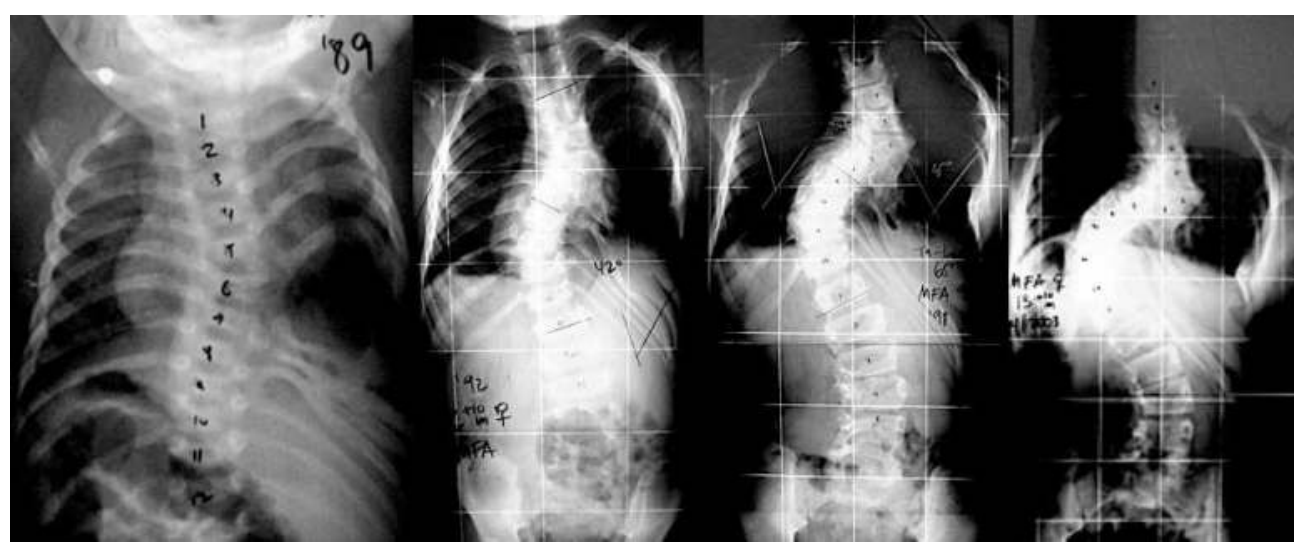

Fig. 1. Natural history of congenital spinal deformity from the newborn period to 13 years of age.

Not been defined the best surgical treatment for hemivertebra deformity. Surgical treatments described include: "in situ" fusion, convex hemiepiphysiodesis and hemiartrhodesis and hemivertebra resection.

The "in situ" fusion and convex hemiepiphysiodesis and hemiartrhodesis have limited effectiveness and unpredictable results in terms of halting the progression and correction of 
the deformity. Resection of the hemivertebra appears as a surgical alternative that allows to stop the progression of the curve and correct the deformity.

\section{Etiology}

Congenital kyphoscoliosis is defined as spinal deformity present at birth due to abnormal vertebral development.

Congenital spine malformations are produced by defects in somitogenesis during the embryonic development comprised between 5 and 8 weeks of gestation (Kusumi \& Turnpenny, 2007). Are frequently associated with cardiac, urologic and respiratory abnormalities in up to $60 \%$ of cases (Basu et al., 2002; Beals et al., 1993), and also to neural axis abnormalities in up to $38 \%$ of cases (Basu et al., 2002; Bradford et al., 1991).

The exact cause of congenital vertebral malformations is unknown, however, many experimental studies in animals suggest that maternal exposure to various noxas (carbon monoxide, smoking, alcohol, valproic acid, etc.) during this critical period of embryonic development can lead to defects in the development of the spine (Hensinger, 2009).

There is no hereditary pattern in the development of vertebral malformations, however, has been identified specific genetic abnormalities which are associated with development of spondylothoracic dysplasia and Alagille syndrome (Bulman et al., 2000; Erol et al., 2004).

Current evidence suggests that both environmental and genetic factors were involved in the development of the spinal malformations.

\section{Classification}

Vertebral congenital anomalies are classified as defects of formation (hemivertebra, wedge vertebra), segmentation defects (bars, block vertebra) and mixed (bar with contralateral hemivertebra).

The hemivertebra is a defect of formation and according to the presence or absence of adjacent intervertebral discs, can be classified as fully segmented, partially segmented or unsegmented (Putti, 1910, as cited in Winter \& Lonstein, 2010) (Figures 2 - 3).

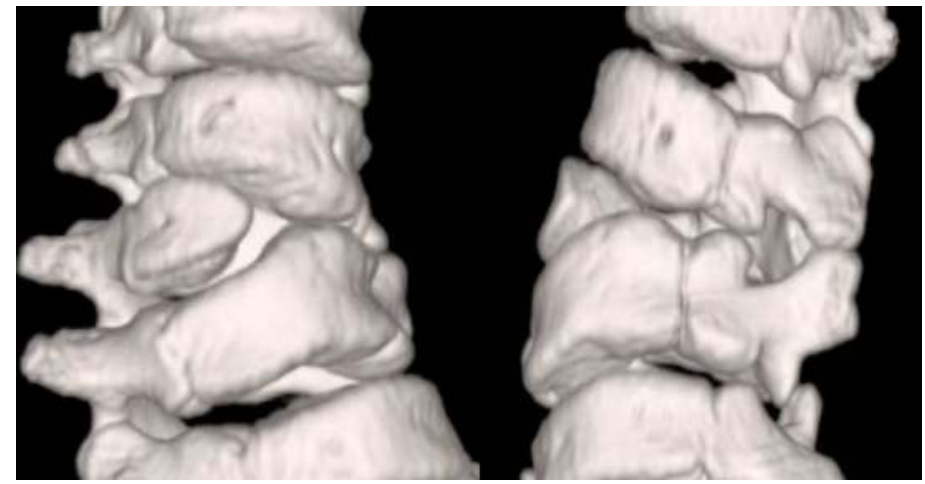

Fig. 2. 3D CT scan reconstruction of fully segmented hemivertebra. 


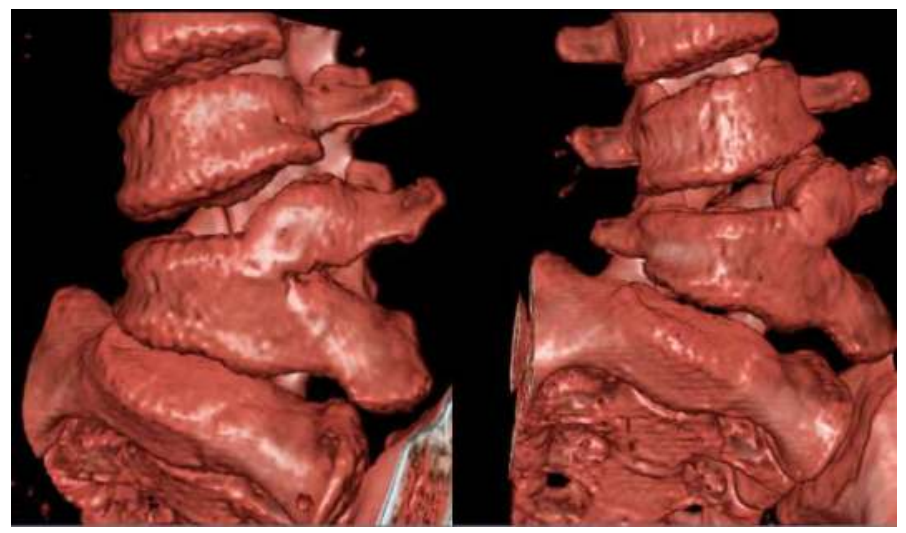

Fig. 3. 3D CT scan reconstruction of partially segmented hemivertebra.

On the other hand, according to its alignment and relationship with the rest of the spine can be classified as "incarcerated", if it maintains the pedicle alignment without protrudes vertebral contour, or as "non incarcerated", if it loses the pedicle alignment and protrudes vertebral contour (McMaster \& Ohtsuka, 1982).

\section{Epidemiology}

The exact prevalence of congenital vertebral malformations is unknown but was estimated at 1 per 1000 live births (Giampietro et al., 2003). The incidence of hemivertebra reaches $0.125 \%$ at 12000 antenatal ultrasonography (Freeman et al., 2001).

The deformity secondary to vertebral malformation can alter the sagittal and coronal plane, being congenital kyphosis and lordosis much less common than congenital scoliosis, which includes $80 \%$ of cases (McMaster \& Ohtsuka, 1982; Winter et al., 1968).

The hemivertebra is the most common spinal malformation present in $35 \%$ to $45 \%$ of congenital kyphoscoliosis (McMaster \& Ohtsuka, 1982; Mohanty \& Kumar, 2000).

In relation to the types of hemivertebra, McMaster \& David, in their study of 154 hemivertebra found $65 \%$ fully segmented hemivertebra, $22 \%$ partially segmented and $12 \%$ unsegmented (McMaster \& David, 1986).

\section{Natural history}

The natural history of congenital kyphoscoliosis is well known, however, due to the large variability of vertebral malformations present in the congenital kyphoscoliosis is not possible to accurately predict the risk of progression. Several studies conclude that $25 \%$ of congenital kyphoscoliosis not progress, $25 \%$ presents moderate progress and $50 \%$ presents severe progress requiring surgical resolution (McMaster \& Ohtsuka, 1982).

The degree of scoliosis produced by hemivertebra depends on following 4 factors (McMaster \& David, 1986):

Type of hemivertebra: segmented and non incarcerated hemivertebra progress to most severe deformities. 
Location: location of the hemivertebra in transitional zones between mobile segments and less mobile or fixed segments (cervicothoracic, thoracolumbar and lumbosacral) determines a greater trunk imbalance and clinically visible deformities, developing shoulders asymmetry and pelvic obliquity in cases of high thoracic and lumbosacral hemivertebra, respectively (Figure 4).

Number of hemivertebra and its relationship: contralateral hemivertebra in the same area of the spine tend to generate compensatory curves and less severe deformities unlike hemivertebra located in different areas of the spine and hemivertebra located ipsilaterally (Figure 5).

Age of patient: the first 5 years of life and puberty are the 2 periods of rapid growth of the spine where the deformity may progress rapidly (Dimeglio, 2001).

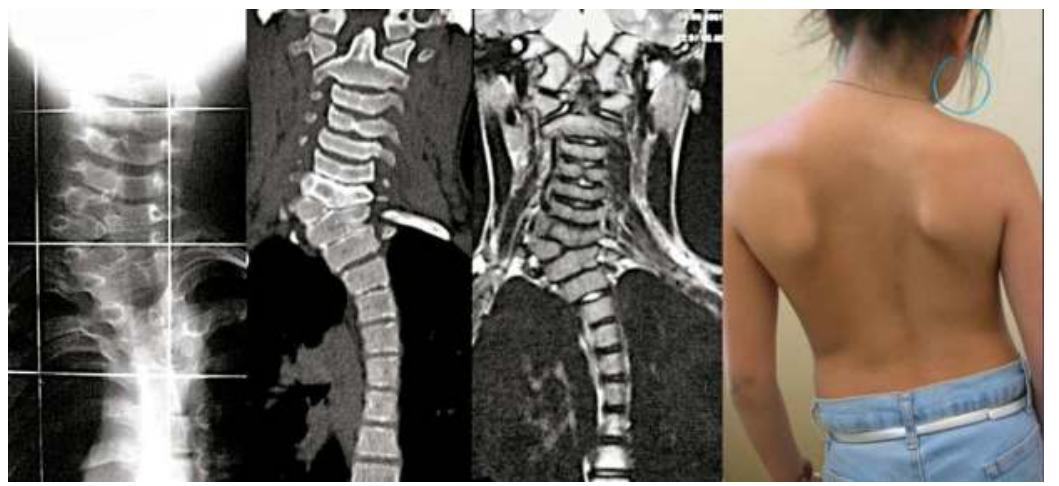

Fig. 4.12 year old girl with T1 hemivertebra, note the important deformity and trunk imbalance due to his location in cervicothoracic transitional zone.

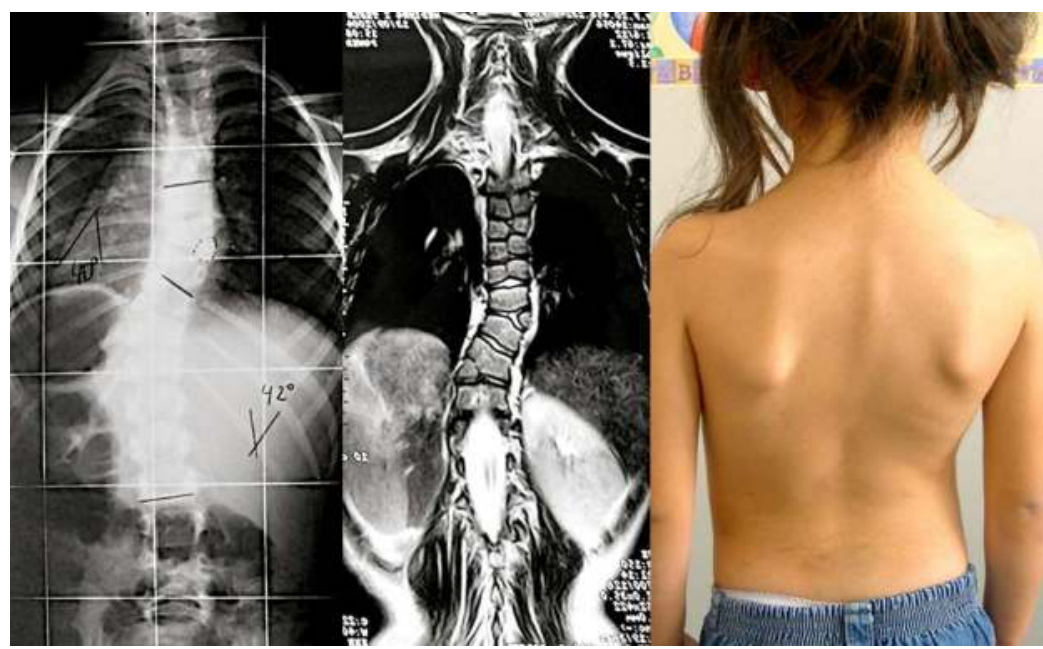

Fig. 5.5 year old girl with balanced congenital deformity, multiple butterfly vertebra in the upper toracic spine and opposite side hemivertebra in T9 and T11. 
The hemivertebra associated with contralateral bar has the worst prognosis of progression (5 to 10 degrees/year) followed by two unilateral hemivertebra (2 to 5 degrees/year), single fully segmented hemivertebra (1 to 3 degrees/year) and wedge vertebra (1 to 2 degrees/year) (McMaster \& Ohtsuka, 1982) (Table 1).

\begin{tabular}{|l|l|}
\hline \multicolumn{1}{|c|}{ Type of formation failiure } & \multicolumn{1}{c|}{ Rate of progression } \\
\hline Hemivertebra with contralateral bar & 5 to 10 degrees/year \\
\hline Two unilateral hemivertebra & 2 to 5 degrees/year \\
\hline Single fully segmented hemivertebra & 1 to 3 degrees/year \\
\hline Wedge vertebra & 1 to 2 degrees/year \\
\hline
\end{tabular}

Table 1. Formation defects progression.

In relation to specific types of hemivertebrae and curve magnitude at the end of growth, unsegmented incarcerated hemivertebra never progresses beyond 30 degrees, partially segmented hemivertebra progresses to angular values that do not exceed 40 degrees and fully segmented hemivertebra progresses to values higher than 40 degrees.

Studies about natural history of congenital scoliosis due to hemivertebra confirm the progressive course of the deformity, with curves above 40 degrees at skeletal maturity in $64 \%$ to $84 \%$ of cases (McMaster \& Ohtsuka, 1982; McMaster \& David, 1986).

\section{Treatment options}

Each patient with kyphoscoliosis due to hemivertebra is unique, therefore, the choice of treatment will be based on the risk of curve progression during growth.

Conservative treatment, based on observation and brace has proved ineffective in the control of curve progression, often requiring surgical resolution. As mentioned above, most congenital kyphoscoliosis due to hemivertebra require surgery to prevent the development of severe deformities.

The key is the early detection of the deformity and early surgical management to prevent secondary curves progress and avoid to become structural, preventing the development of severe deformities wich requires complex surgeries and extensive spinal fusion.

Classically it describes three surgical procedures: "in situ" fusion, whose aim is to stop curve progression without correction of this, convex hemiepiphysiodesis and hemiartrhodesis, which aims to halt the progression and achieve gradual correction deformity at the time, and finally, hemivertebra resection which aim is to stop the progression, correction of the curve acutely and prevent the development of secondary curves.

\subsection{Conservative treatment}

Periodic monitoring is indicated only in patients with clinical and radiographic balanced and non progressive deformities.

Brace has no place in the treatment of congenital kyphoscolisis, only have some utility in the control of secondary curves in selected cases. 


\subsection{Surgical treatment}

The goals of surgical treatment are to achieve a solid fusion to stop the progression of the curve, and reach the end of growth with a spine properly aligned and well balanced.

There are 2 categories of surgical procedures; prophylactic surgery and corrective surgery. Prophylactic surgeries are intended to prevent progression of curve ("in situ" fusion) or gradual correction of curve across the time (hemiepiphyisiodesis). On the other hand, the corrective procedures aim to correct the deformity and prevent the development of secondary curves (hemivertebra resection).

Surgical treatment should be early and ideally should be performed before 5 years of age.

The incorporation and use of instrumentation in young children, especially the use of pedicle screws, is one of the most important advances in recent years. Its proven efficacy and safety, have made the outcomes of different surgical techniques are progressively better and with fewer complications related to the use of implants (Hedequist et al., 2004; Ruf \& Harms, 2002).

\subsection{1 "In situ" fusion}

Cataloged in prophylactic surgical procedures, "in situ" fusion is the oldest surgical strategy for treatment of congenital kyphoscolisis. It corresponds to fusion of a short spinal segment includes vertebral malformation, preserving spinal mobility without significant compromise on the length of the spine at the end of growth.

Patients without or minimal deformity, but with malformations whose progression is virtually assured (fully segmented hemivertebra, hemivertebra with contralateral bar) are the best candidates for this surgery (McMaster \& David, 1986; Winter \& Moe, 1982).

Classically "in situ" fusion was described by a unique posterior approach without instrumentation associated with the use of corrective brace. Has been described a posterior approach and combined anterior and posterior approach, with or without instrumentation.

The long-term follow-up showed a high rate of progression attributed to the presence of a flexible callus, present in 39\% of patients with remaining growth (Winter \& Moe, 1982), and the crankshaft phenomenon, present in $15 \%$ of total patients and in $36 \%$ of patients younger than 4 years (Kesling et al., 2003).

Patients with anterior growth potential require a anterior or combined approach to prevent the development of the crankshaft phenomenon. In cases of congenital lordosis, anterior fusion is the key to preventing the progression of the deformity, unlike the cases of congenital kyphosis, where the exclusive posterior approach and posterior fusion preserving the anterior spinal growth allow gradual correction of the deformity (Winter et al., 1996).

The use of instrumentation has been shown to improve fusion rates and the degree of curve correction, with a low rate of complications associated with the use of implants (Hedequist et al., 2004), however, this does not result in a significant improvement in long-term clinical outcomes (Farley et al., 2011). 
The results of this surgical strategy remain unpredictable with incidence rates of progression after surgery ranging from $11 \%$ to $42 \%$ of cases. Goldberg et al. indicate that of 43 patients underwent fusion "in situ", 25.6\% required reoperation for unacceptable progression of curve (Goldberg et al., 2002), a rate that does not differ from the rate of surgeries presented in natural history studies of Mc Master (McMaster \& Ohtsuka, 1982; McMaster \& David, 1986), and suggests that this procedure would be ineffective in the management of congenital kyphoscoliosis, and it does not modify the natural history of the disease.

Today this technique is practically abandoned because of the inability to control the progression of the curve, the high rate of reoperations and poor clinical outcomes in the long-term follow-up.

\subsubsection{Convex hemiepiphysiodesis and hemiartrhodesis}

Considered in prophylactic surgery, the goal is to halt the growth of the convexity of the curve and maintain growth of the concavity achieving theoretically the gradual correction of the deformity. It is imperative that the patient have growth potential in the concavity of curve, therefore that surgery is reserved for formation vertebral defects and should be performed early, before 5 years of age.

Is recommended with curves under 50 degrees, because curves greater than 50 degrees have higher incidence of fusion.

The classic indication is patient with fully segmented hemivertebra without other spinal malformation.

Requires combined anterior and posterior approaches to prevent development of deformities in the sagittal plane. The anterior hemiepiphysiodesis involves removing $1 / 3$ of the intervertebral disc and its corresponding vertebral endplates and fuse them, and the posterior hemiarthrodesis involves the exclusive convex side exposure and its fusion with or without instrumentation (Shen, F. \& Arlet, V. 2008).

The anterior hemiepiphysiodesis may be made by classic combined open anterior and posterior approaches, endoscopic or transpedicular posterior approach.

We do not recommend this technique when deformity have a major kyphotic component, because the growth of the posterior elements of the concavity may generate significant progression of the deformity in the sagittal plane in 36\% of cases (Cil et al., 2004).

Uzumcugil et al. in a review of different series of patients treated with convex hemiepiphysiodesis and hemiarthrodesis notes that the hemiepiphisiodesis effect is achieved in $20 \%$ to $77 \%$ of cases, fusion effect in $17 \%$ to $70 \%$ and progression in the $0 \%$ to $21 \%$, demonstrating that this technique results are unpredictable (Uzumcugil et al., 2004). In cases where hemiepiphisiodesis effect is achieved, the average curve correction does not exceed 15 to 20 degrees (Winter \& Lonstein, 1988).

The overall results of this technique are variable and unpredictable, however, this technique has a low complication rate, virtually only related to anterior approaches. 


\subsubsection{Hemivertebra resection}

The first report of resection of hemivertebra was made by Royle in 1928. Despite the high rate of complications and initial technical difficulties, has gradually incorporated as a valid surgical alternative.

The aim is to remove the source of the deformity, correction of the curve and prevent the development of secondary curves.

Currently considered the method of choice for the treatment of congenital kyphoscoliosis secondary to isolated hemivertebra.

The best indication of hemivertebra resection is: patients younger than 5 years with thoracolumbar, lumbar or lumbosacral hemivertebra associated to initial trunk imbalance.

Classically hemivertebra resection was described by combined anterior and posterior approach in 1 or 2 stages. Also have been reported simultaneous anterior/posterior approaches.

Posterior exclusive approaches have become popular. Traditional or classic vertebral resection or eggshell techinque are the surgical options described for the posterior vertebral excision (Figure $6-7$ ).

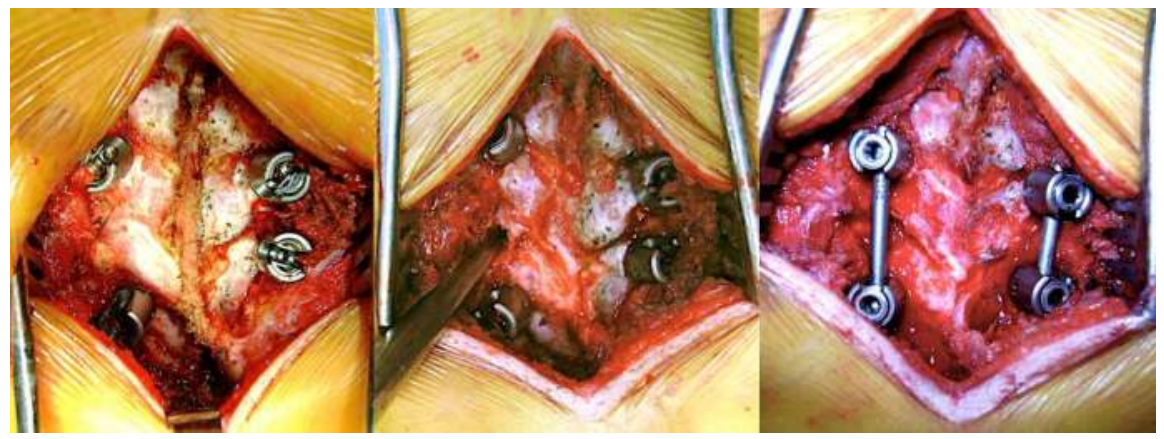

Fig. 6. Eggshell technique.



Fig. 7. 3D CT scan reconstruction of hemivertebra resection with eggshell procedure. 
Initially, to close the gap left by the resected hemivertebra were applied corrective casts and brace for a 6 months period. Then posterior instrumentation was introduced, which allowed the compression of the convexity using wires, hooks and rods. Finally, the incorporation and use of pedicle screws in young children has made more rigid and shorter fixations, allowing monosegmental pedicular instrumentation with good outcomes at medium-term follow-up. (Ruf \& Harms, 2003; Ruf et al., 2009).

There are also some reports of series where anterior instrumentation was used with good results in the short-term follow-up (Elsebaie et al., 2010; Garrido et al., 2008).

The operating time of anterior and posterior combined approach that goes from 280 to 435 minutes, slightly above the average operating time exclusive posterior approach, ranging from 225 to 380 minutes (Arriagada et al., 2009).

Holte et al. in a series of hemivertebra resection by combined approach showed overall complication rate of $65 \%$ with $21 \%$ of neurological complications (Holte et al., 1995). Over time, the overall complication rate decreased to values from $0 \%$ to $30 \%$, with $5 \%$ to $10 \%$ of neurological complications, particularly transient neurological injury.

Recent research indicates that complication rates associated to the exclusive posterior approach $(14 \%)$ is slightly higher than the combined approach $(9 \%)$, without significant differences in relation to neurological complications (Jalanko et al., 2010).

Combined approaches are mainly associated with medical complications derived from anterior approaches (thoracotomy) and posterior approaches are mainly associated with complications derived from implant failure and the need for reoperations.

The global analysis of hemivertebra resections, regardless of approach, shows average correction of $35 \%$ to $81 \%$ in the coronal plane and $8 \%$ to $79 \%$ in the sagittal plane, with a minimum follow-up of 2 years.

In the short and medium-term follow-up, surgeries perfomed through combined approach allow corrections of scoliosis from $35 \%$ to $77 \%$, without significant differences with respect to the exclusive posterior approach, which allows correction of $27 \%$ to $81 \%$ (Table 2).

\begin{tabular}{|l|c|c|c|c|c|c|}
\hline Approach & \multirow{2}{*}{ Instrument. } & \multicolumn{2}{|c|}{ Correction (\%) } & \multirow{2}{*}{ Operative time (min) } & EBL (ml) & Complic. (\%) \\
\cline { 3 - 4 } & & Scoliosis & Kyphosis & & & \\
\hline Posterior & Posterior & $27-81$ & $33-80$ & $225-380$ & $114-885$ & $8-40$ \\
\hline \multirow{2}{*}{ Combined } & Anterior & $56-64$ & $31-49$ & $120-133$ & $150-169$ & $8-13$ \\
\cline { 2 - 7 } & Posterior & $35-77$ & $32-42$ & $280-435$ & $280-2767$ & $8-38$ \\
\hline
\end{tabular}

Table 2. Results of hemivertebra resection strategies.

The use instrumentation in the exclusive posterior approach with unilateral or bilateral monosegmental fixation is associated with frequent implant failures and the need for additional surgeries. The use of a third bar or the use of postoperative brace allows to reduce that risk.

The traditional or classic vertebral resection by exclusive posterior approach, regardless instrumentation was used, achieved corrections above $40 \%$ with minimal loss of correction during long-term follow-up. By contrast, vertebral resection with eggshell technique shown 
corrections of $27 \%$ in the medium-term follow-up, with a loss of scoliosis correction of $25 \%$ (Table 3).

\begin{tabular}{|c|c|c|c|c|c|}
\hline \multirow{2}{*}{ Posterior Approach } & \multicolumn{2}{|c|}{ Correction (\%) } & \multirow{2}{*}{ Operative time (min) } & \multicolumn{2}{c|}{ Complic. (\%) } \\
\cline { 2 - 3 } \cline { 5 - 6 } & Scoliosis & Kyphosis & & Total & Neuro \\
\hline Classic & $54-81$ & $50-80$ & $225-380$ & $8-40$ & $0-10$ \\
\hline Eggshell & 27 & 28 & 225 & 29 & 0 \\
\hline
\end{tabular}

Table 3. Results posterior approach: classic and eggshell technique.

The growth potential of patients and the use of monosegmental fixations preserving unfused levels makes the progression and loss of correction is an expected and predictable phenomenon. Apparently eggshell resection would not allow such accurate and complete removal of adjacent intervertebral discs as well not proper preparation of adjacent vertebral plates to provide adequate anterior fusion. Furthermore, pedicle instrumentation monosegmental not be able to slow the growth of the hemivertebra above, allowing the progression of the curves.

With the eggshell technique produces better results than the "in situ" fusion and convex hemiepiphysiodesis in terms of correction and control the progression of the curve, with a similar complication rates, so that this technique appears as an intermediate alternative between these 2 strategies and complete resection of the hemivertebra, achieving acceptable and predictable angular correction, avoiding the potentially serious complications of complete vertebral resection.

For all these reasons we believe that, although the eggshell technique allows minor corrections that technique of complete resection of hemivertebra, remains a safe and less invasive alternative that can be used in a special group of patients who require surgery with low risk and predictable results, even when the magnitude of these results is less than those obtained with the complex vertebral resection by combined or single posterior approach.

\section{Conclusion}

The spinal malformations arise from defects in very early stages of embryonic development, due to this presents a wide variety of vertebral anomalies and their frequent association with malformations in other systems (cardiac, urologic, respiratory and neural axis).

Numerous studies of the natural history of congenital spinal deformities due to hemivertebra are categorical in the progressive nature of these deformities. It is therefore important to define accurately the type of malformation detected in the patient and determine the potential risk of progression. The most common spinal defect is the hemivertebra, which causes about $50 \%$ of cases of congenital kyphoscoliosis.

The ideal treatment for hemivertebra deformity should be able to correct the deformity, stop curve progression and prevent the development of structured secondary curves, with low complication rate.

It is demonstrated that the conservative treatment and use of brace has no place in the management of congenital kyphoscoliosis, therefore the treatment is mainly surgical. 
Strict controls of patients, to objectify the evolution of the deformity is vital to define a possible surgical treatment at the right time.

Young children with deformities which have a guaranteed progression and demonstrate a curve progression with clinical repercussion are candidates for surgery. To achieve the best results, we recommend surgical treatment within 5 years of age.

Has been described three surgical options for management of kyphoscoliosis due to hemivertebra: "in situ" fusion, convex hemiepiphysiodesis and hemiartrhodesis and hemivertebra resection.

The literature review shows us that the in situ fusion is a procedure incapable to correct and control the progression of the curve, without changing the natural course of disease, for this reason its use has been virtually abandoned today.

On the other hand, the convex hemiepiphysiodesis and hemiarthrodesis is a safe procedure that shows some effectiveness in terms of correction and control of the progression, but the results are completely unpredictable in time.

Finally, resection of hemivertebra enables setting an adequate and predictable correction of the curve and stop progression, but is associated with a considerable number of complications inherent to surgery or related to use of implants, which can be potentially severe.

For all the above, there is no ideal treatment for this condition, each individual case must be analyzed, carefully assessing the condition of patient, potential risks and expected outcomes.

\section{Acknowledgment}

To our families, friends and colleagues.

\section{References}

Arriagada, G., Chahin, A., Pantoja, S \& Chamorro, M. (2009). Hemivertebrae Resection with Eggshell Procedure for Congenital Kyphoscoliosis: Results in Children Aged From One to Seven Years. Coluna/Columna. Vol.8, No.3, (July/September 2009), pp. 337343, ISSN 1808-1851

Basu, P., Elsebaie, H. \& Noordeen, M. (2002). Congenital spinal deformity: a comprehensive assessment at presentation. Spine. Vol.27, No.20, (October 2002), pp. 2255-2259, ISSN 1528-1159

Beals, R., Robbins, J. \& Rolfe, B. (1993). Anomalies associated with vertebral malformations. Spine. Vol.18, No.10, (August 1993), pp. 1329-1332, ISSN 1528-1159

Bradford, D., Heithoff, K. \& Cohen M. (1991). Intraspinal abnormalities and congenital spine deformities: A radiographic and MRI study. Journal of Pediatric Orthopaedics. Vol.11, No.1, (January/February 1991), pp. 36-41, ISSN 1539-2570 
Bulman, M., Kusumi, K. \& Frayling, T. (2000). Mutations in the human delta homologue, DLL3, cause axial skeletal defects in spondylocostal dysostosis. Nature Genetics. Vol.24, No.4, (April 2000), pp. 438-441, ISSN 1546-1718

Cil, A., Yazici, M., Alanay, M., Acaroglu, E., Uzumcugil, A. Surat, A. (2004). The Course of Sagittal Plane Abnormality in the Patients With Congenital Scoliosis Managed With Convex Growth Arrest. Spine. Vol.29, No.5, (March 2004), pp. 547-553, ISSN 15281159

Dimeglio, A. (2001). Growth in pediatric orthopedics. Journal of Pediatric Orthopaedics. Vol.21, No.4, (July/August 2001), pp. 549-555, ISSN 1539-2570

Erol, B., Tracy, M., Dormans, J., Zackai, E., Maisenbacher, M., O’Brien, M., Turnpenny, P. \& Kusumi, K. (2004). Congenital scoliosis and vertebral malformations: characterization of segmental defects for genetic analysis. Journal of Pediatric Orthopaedics. Vol.24, No.6, (November/December 2004), pp. 674-682, ISSN 15392570

Farley, F., Have, K., Hensinger, R., Streit, J., Zhang, L. \& Caird, M. (2011). Outcomes After Spinal Fusion for Congenital Scoliosis Instrumented Versus Uninstrumented Spinal Fusion. Spine. Vol.36, No.2, (January 2011), pp. E112E122, ISSN 1528-1159

Freeman, B., Ouellet, J., Twining, P. \& Webb, J. (2001). Antenatal diagnosis and ultimate outcome of fetal hemivertebrae. Scoliosis Research Society annual meeting 2001; http://www.spineuniverse.com/professional/research/srs/2001/antenataldiagnosis-ultimate-outcome-fetal

Giampietro PF, Blank RD, Raggio CL, et al. (2003). Congenital and idiopathic scoliosis: clinical and genetic aspects. Clinincal Medicine E Research. Vol.1, No.2, (April 2003), pp. 125-136, ISSN 1554-6179

Goldberg, C., Moore, D., Fogarty, E. \& Dowling, F. (2002). Long-term results from in situ fusion for congenital vertebral deformity. Spine. Vol.27, No.6, (March 2002), pp. 619-628, ISSN 1528-1159

Hedequist, D., Hall, J. \& Emans, J. (2004). The safety and efficacy of spinal instrumentation in children with congenital spine deformities. Spine. Vol.29, No.18, (September 2002), pp. 2081-2086, ISSN 1528-1159

Hedequist, D., Emans, J., Proctor, M. (2009). Three rod technique facilitates hemivertebra wedge resection in young children through a posterior only approach. Spine. Vol.34, No.6, (March 2009), pp. E225-E229, ISSN 1528-1159

Hensinger, R. (2009). Congenital scoliosis: etiology and associations. Spine. Vol.34, No.17, (August 2009), pp. 1745-1750, ISSN 1528-1159

Holte, D., Winter, R., Lonstein, J. \& Denis, F. (1995). Excision of hemivertebrae and wedge resection in the treatment of congenital scoliosis. The Journal of Bone and Joint Surgery Am. Vol.77, No. 2, (February 1995), pp. 159-171, ISSN 1535-1386

Kesling, K., Lonstein, J., Denis, F., Perra, J., Schwender, J. Transfeldt, E. \& Winter, R. (2003). The crankshaft phenomenon after posterior spinal arthrodesis for congenital scoliosis: a review of 54 patients. Spine. Vol.28, No.3, (February 2003), pp. 267-271, ISSN 1528-1159 
Kusumi, K. \& Turnpenny, P. (2007). Formation errors of the vertebral column. The Journal of Bone and Joint Surgery Am. Vol.89, Supplement 1, (February 2007), pp. 64-71, ISSN $1535-1386$

McMaster, M. \& Ohtsuka, K. (1982). The natural history of congenital scoliosis: A study of two hundred and fifty-one patients. The Journal of Bone and Joint Surgery Am. Vol.64, No.8, (October1982), pp. 1128-1147, ISSN 1535-1386

McMaster, M. \& David C. (1986). Hemivertebra as a cause of scoliosis: a study of 104 patients. The Journal of Bone and Joint Surgery Br. Vol.68-B, No.4, (August 1986), pp. 588-595, ISSN 0301-620X

McMaster, M. \& Singh, H. (1999). Natural history of congenital kyphosis and kyphoscoliosis. A study of one hundred and twelve patients. The Journal of Bone and Joint Surgery Am. Vol.81, No.10, (October1982), pp. 1367-1383, ISSN 15351386

Mohanty, S. \& Kumar, N. (2000). Patterns of presentation of congenital scoliosis. Journal of Orthopeadic Surgery. Vol.8, No.2, (December 2000), pp. 33-37, ISSN 1022-5536

Nasca, R., Stelling, F. \& Steel, H. (1975). Progression of congenital scoliosis due to hemivertebrae and hemivertebrae with bars. The Journal of Bone and Joint Surgery Am. Vol.57, No.4, (June 1975), pp. 456-466, ISSN 1535-1386

Ruf, M. \& Harms, J. (2002). Pedicle screws in one and two year old children: Technique, complications, and effect on further growth. Spine. Vol.27, No.21, (November 2002), pp. E460-E466, ISSN 1528-1159

Ruf, M. \& Harms, J. (2003). Posterior hemivertebra resection with transpedicular instrumentation: early correction in children aged 1 to 6 years. Spine. Vol.28, No.18, (September 2003), pp. 2132-2138, ISSN 1528-1159

Ruf, M., Jensen, R., Letko, L. \& Harms, J. (2009). Hemivertebra resection and osteotomies in congenital spine deformity. Spine. Vol.34, No.17, (August 2009), pp. 1791-1799, ISSN 1528-1159

Shen, F. \& Arlet, V. (2008). Congenital Scoliosis, In: Spinal Disorders. Fundamentals of Diagnosis and Treatment, Boos, N. \& Aebi (Ed.), M., pp. 693-711, Springer, ISBN 9783-540-40511-5, Germany

Uzumcugil, A., Cil, A., Yazici, M., Acaroglu, E., Alanay, A., Aksoy, C. \& Surat, A. (2004). Convex growth arrest in the treatment of congenital spinal deformities, revisited. Journal of Pediatric Orthopaedics. Vol.24, No.6, (November/December 2004), pp. 658666, ISSN 1539-2570

Winter, R., Moe, J. \& Eilers, V. (1968). Congenital scoliosis: A study of 234 patients treated and untreated: Part I. Natural history. The Journal of Bone and Joint Surgery Am. Vol.50 , No. 1, (January 1968), pp. 1-15, ISSN 1535-1386

Winter, R. \& Moe, J. (1982). The results of spinal arthrodesis for congenital spinal deformity in patients younger than five years old. The Journal of Bone and Joint Surgery Am. Vol.64 , No. 3, (March 1982), pp. 419-432, ISSN 1535-1386

Winter, R., Lonstein, J. Denis, F. \& de la Rosa, H. (1988). Convex growth arrest for progressive congenital scoliosis due to hemivertebrae. Journal of Pediatric Orthopaedics. Vol.8, No.6, (November 1988), pp. 633-638, ISSN 1539-2570 
Winter, R., Lonstein, J. \& Boachie-Adjei, O. (1996). Instructional Course Lectures, The American Academy of Orthopaedic Surgeons: Congenital Spinal Deformity. The Journal of Bone and Joint Surgery Am. Vol.78-A, No. 2, (February 1996), pp. 300-311, ISSN 1535-1386

Winter, R. \& Lonstein, J. (2010). Scoliosis Secondary to a Hemivertebra. Seven Patients With Gradual Improvement Without Treatment. Spine. Vol.35, No.2, (January 2010), pp. E49-E52, ISSN 1528-1159 


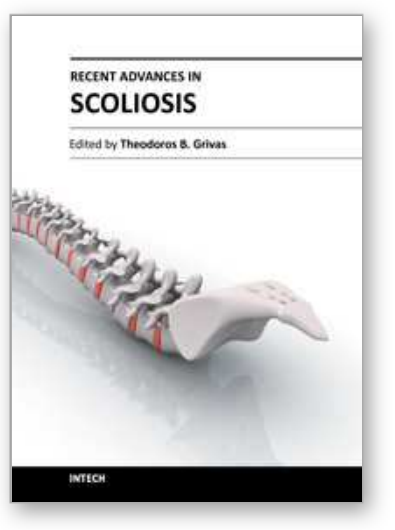

\author{
Recent Advances in Scoliosis \\ Edited by Dr Theodoros Grivas
}

ISBN 978-953-51-0595-4

Hard cover, 344 pages

Publisher InTech

Published online 09, May, 2012

Published in print edition May, 2012

This book contains information on recent advances in aetiology and pathogenesis of idiopathic scoliosis, for the assessment of this condition before treatment and during the follow-up, making a note of emerging technology and analytical techniques like virtual anatomy by 3-D MRI/CT, quantitative MRI and Moire Topography. Some new trends in conservative treatment and the long term outcome and complications of surgical treatment are described. Issues like health related quality of life, psychological aspects of scoliosis treatment and the very important "patient's perspective" are also discussed. Finally two chapters tapping the untreated early onset scoliosis and the congenital kyphoscoliosis due to hemivertebra are included. It must be emphasized that knowledgeable authors with their contributions share their experience and enthusiasm with peers interested in scoliosis.

\title{
How to reference
}

In order to correctly reference this scholarly work, feel free to copy and paste the following:

Andrés Chahin Ferreyra and Gonzalo Arriagada Ocampo (2012). Congenital Kyphoscoliosis Due to Hemivertebra Treatment Options and Results, Recent Advances in Scoliosis, Dr Theodoros Grivas (Ed.), ISBN: 978-953-51-0595-4, InTech, Available from: http://www.intechopen.com/books/recent-advances-inscoliosis/congenital-kyphoscoliosis-due-to-hemivertebra-treatment-options-and-results

\section{INTECH}

open science | open minds

\author{
InTech Europe \\ University Campus STeP Ri \\ Slavka Krautzeka 83/A \\ 51000 Rijeka, Croatia \\ Phone: +385 (51) 770447 \\ Fax: +385 (51) 686166 \\ www.intechopen.com
}

\author{
InTech China \\ Unit 405, Office Block, Hotel Equatorial Shanghai \\ No.65, Yan An Road (West), Shanghai, 200040, China \\ 中国上海市延安西路65号上海国际贵都大饭店办公楼405单元 \\ Phone: +86-21-62489820 \\ Fax: +86-21-62489821
}


(C) 2012 The Author(s). Licensee IntechOpen. This is an open access article distributed under the terms of the Creative Commons Attribution 3.0 License, which permits unrestricted use, distribution, and reproduction in any medium, provided the original work is properly cited. 